\title{
A modified complex short pulse equation of defocusing type
}

Shoufeng Shen, Bao-Feng Feng, Yasuhiro Ohta

To cite this article: Shoufeng Shen, Bao-Feng Feng, Yasuhiro Ohta (2017) A modified complex short pulse equation of defocusing type, Journal of Nonlinear Mathematical Physics 24:2, 195-209, DOI: https://doi.org/10.1080/14029251.2017.1306946

To link to this article: https://doi.org/10.1080/14029251.2017.1306946

Published online: 04 January 2021 


\title{
A modified complex short pulse equation of defocusing type
}

\author{
Shoufeng Shen* \\ Department of Applied Mathematics, Zhejiang University of Technology \\ Hangzhou, 310023, China \\ mathssf@zjut.edu.cn \\ Bao-Feng Feng \\ School of Mathematical and Statistical Sciences, The University of Texas Rio Grande Valley \\ TX, 78541, USA \\ Yasuhiro Ohta \\ Department of Mathematics, Kobe University \\ Rokko, Kobe 657-8501, Japan
}

Received 26 August 2016

Accepted 11 February 2017

\begin{abstract}
In this paper, we are concerned with a modified complex short pulse (mCSP) equation of defocusing type. Firstly, we show that the mCSP equation is linked to a complex coupled dispersionless equation of defocusing type via a hodograph transformation, thus, its Lax pair can be deduced. Then the bilinearization of the defocusing mCSP equation is formulated via dependent variable and hodograph transformations. One- and two-dark soliton solutions are found by Hirota's bilinear method and their properties are analyzed. It is shown that, depending on the parameters, the dark soliton solution can be either smoothed, cusponed or looped one. More specifically, the dark soliton tends to be evolved into a singular (cusponed or looped) one due to the increase of the spatial wave number in background plane waves and the increase of the depth of the trough. In the last part of the paper, we derive the defocusing mCSP equation from the single-component extended KP hierarchy by the reduction method. As a by-product, the $N$-dark soliton solution in the form of determinants for the defocusing $\mathrm{mCSP}$ is provided.
\end{abstract}

Keywords: Short pulse equation; hodograph transformation; dark soliton; Hirota's bilinear method.

2000 Mathematics Subject Classification: 35Q53

\section{Introduction}

The so-called short pulse (SP) equation

$$
u_{x t}=u+\frac{1}{6}\left(u^{3}\right)_{x x},
$$

was proposed by Schäfer and Wayne to describe the propagation of ultra-short optical pulses in nonlinear media $[4,30]$. Here, $u=u(x, t)$ is a real-valued function, representing the magnitude of the electric field, the subscripts $t$ and $x$ denote partial differentiation. Apart from the context of nonlinear optics, the SP equation has also been derived as an integrable differential equation associated with pseudospherical surfaces $[1,25]$. The SP equation has been shown to be completely integrable

$\overline{{ }^{*} \text { Corresponding author. }}$ 
possessing a Lax pair representation and bi-Hamiltonian structure $[2,3,26]$. The connection between the SP equation and the sine-Gordon equation through the reciprocal transformation was firstly discovered in [26], then was further clarified and used by to find two-loop and breather solutions in [27] by the same authors. The general $N$-soliton solutions including multi-loop and multi-breather ones were given in [19] by using Hirota's bilinear method [14,18]. The integrable discretization and the geometric interpretation of the SP equation were given in $[6,7]$.

Recently, a modified short pulse (mSP) equation

$$
u_{x t}=u+u u_{x}^{2}+u^{2} u_{x x},
$$

was studied by Sakovich [28], in which the Lax pair and the soliton solutions were provided. The $\mathrm{mSP}$ equation is a direct reduction $(u=v)$ of a coupled short pulse equation [8] proposed by one of the authors

$$
u_{x t}=u+\frac{1}{6}\left(u^{3}\right)_{x x}+\frac{1}{2} v^{2} u_{x x}, \quad v_{x t}=v+\frac{1}{6}\left(v^{3}\right)_{x x}+\frac{1}{2} u^{2} v_{x x} .
$$

Besides the above coupled short pulse equation (1.3), there is another coupled pulse equation proposed by Müller-Hoissen and Matsuno independently [5,20]

$$
u_{x t}=u+\frac{1}{2}\left(u v u_{x}\right)_{x}, v_{x t}=v+\frac{1}{2}\left(u v v_{x}\right)_{x} .
$$

When $v^{*}=u$, which means $u$ is the complex conjugate of $v$, this leads to a complex short pulse equation

$$
u_{x t}=u+\frac{1}{2}\left(|u|^{2} u_{x}\right)_{x},
$$

which can be derived from the Maxwell equations for the propagation of ultra-short pulses $[10,16]$. The geometric interpretation via the motion of space curves was given in [31]. Its various solutions including the multi-bright soliton, multi-breather and multi-rogue wave solutions were investigated by the Darboux transformation [17].

Quite recently, Matsuno presents a multi-component generalization of the mSP equation [21], of which the two-component mSP equation takes the form

$$
u_{x t}=u+v\left(u u_{x}\right)_{x}, \quad v_{x t}=v+u\left(v v_{x}\right)_{x} .
$$

Especially, imposing $v^{*}=u(\equiv q)$, the two-component mSP equation reduces to a single equation of complex version

$$
q_{x t}=q+q^{*}\left(q q_{x}\right)_{x},
$$

which can be called the modified complex short pulse (mCSP) equation. Similar to the complex short pulse equation which admits both the focusing and defocusing type [11,12], it is natural to consider a modified complex short pulse equation of defocusing type

$$
q_{x t}=q-q^{*}\left(q q_{x}\right)_{x} .
$$

Note that the equation (1.8) is invariant under the following transformation

$$
q \rightarrow c q, \quad \partial_{x} \rightarrow \frac{1}{c} \partial_{x}, \quad \partial_{t} \rightarrow c \partial_{t},
$$

therefore, without loss of generality, the amplitude of $q$ can be fixed as $x \rightarrow \pm \infty$. 
The rest of the paper is organized as follows. In Section 2, the bilinearization of the defocusing mCSP equation (1.8) is formulated via an appropriate hodograph transformation, and the Lax pair is given based on its connection with the complex dispersionless equation of defocusing type. Then the one- and two-dark soliton solutions are found and illustrated, their properties are analysed in details. In Section 3, starting from a single-component extended KP hierarchy, along with its tau functions, the defocusing mCSP equation is derived and its multi-dark soliton solution in determinant form is provided. The paper is concluded by Section 4 .

\section{Integrability of the defocusing mCSP equation}

\subsection{Bilinearization}

The defocusing mCSP equation (1.8) can be deduced by the following bilinear equations

$$
\begin{gathered}
\left(D_{y} D_{s}+\mathrm{i} \kappa D_{s}+\mathrm{i} \gamma D_{y}\right) g \cdot f=0, \\
\left(D_{s}^{2}-\frac{1}{2}\right) f \cdot f=-\frac{1}{2} g g^{*},
\end{gathered}
$$

by means of the dependent variable transformation

$$
q=\frac{1}{2} \frac{g}{f} e^{\mathrm{i}(\kappa y+\gamma s)},
$$

and the hodograph transformation

$$
x=-\kappa \gamma y+\frac{1}{4} s-(\ln f)_{s}, \quad t=s,
$$

where $D$ is the Hirota $D$-operator defined by

$$
D_{s}^{n} D_{y}^{m} f \cdot g=\left.\left(\frac{\partial}{\partial s}-\frac{\partial}{\partial s^{\prime}}\right)^{n}\left(\frac{\partial}{\partial y}-\frac{\partial}{\partial y^{\prime}}\right)^{m} f(y, s) g\left(y^{\prime}, s^{\prime}\right)\right|_{y=y^{\prime}, s=s^{\prime}} .
$$

The proof is given as follows. Dividing $f^{2}$ on both sides of (2.1) and (2.2), and referring to a bilinear identity

$$
\frac{D_{y} D_{s} g \cdot f}{f^{2}}=\left(\frac{g}{f}\right)_{y s}+2(\ln f)_{y s} \frac{g}{f}
$$

we have

$$
\begin{gathered}
\left(\frac{g}{f}\right)_{y s}+2(\ln f)_{y s} \frac{g}{f}+\mathrm{i} \kappa\left(\frac{g}{f}\right)_{s}+\mathrm{i} \gamma\left(\frac{g}{f}\right)_{y}=0, \\
(\ln f)_{s s}=\frac{1}{4}\left(1-\frac{g g^{*}}{f^{2}}\right) .
\end{gathered}
$$

From the hodograph transformation (2.4) and Eq. (2.6), we have

$$
\frac{\partial x}{\partial y}=-\kappa \gamma-(\ln f)_{y s},
$$




$$
\frac{\partial x}{\partial s}=\frac{1}{4}-(\ln f)_{s s}=\frac{1}{4} \frac{g g^{*}}{f^{2}}=|q|^{2}
$$

which implies

$$
\partial_{y}=\rho \partial_{x}, \quad \partial_{s}=\partial_{t}+|q|^{2} \partial_{x},
$$

by defining $\rho=-\kappa \gamma-(\ln f)_{y s}$. Next, we note that Eq. (2.5) can be rearranged as

$$
\left(\frac{g}{f} e^{\mathrm{i}(\kappa y+\gamma s)}\right)_{y s}=\left(-\kappa \gamma-2(\ln f)_{y s}\right) \frac{g}{f} e^{\mathrm{i}(\kappa y+\gamma s)} .
$$

which simply becomes

$$
q_{y s}=(2 \rho+\kappa \gamma) q .
$$

Differentiating Eq. (2.6) with respect to $y$, one obtains

$$
\rho_{s}=\left(|q|^{2}\right)_{y} .
$$

From Eqs. (2.9) and (2.10), one has

$$
\begin{aligned}
\left(q_{y} q_{y}^{*}\right)_{s} & =(2 \rho+\kappa \gamma)\left(q q^{*}\right)_{y} \\
& =(2 \rho+\kappa \gamma) \rho_{s}=\frac{1}{4}\left((2 \rho+\kappa \gamma)^{2}\right)_{s},
\end{aligned}
$$

which implies a conserved quantity

$$
\frac{1}{4}(2 \rho+\kappa \gamma)^{2}-\left|q_{y}\right|^{2}
$$

for the defocusing mCSP equation (1.8). By setting boundary conditions

$$
\left|q_{y}\right| \rightarrow 0, \quad \rho \rightarrow-\kappa \gamma
$$

as $y \rightarrow \pm \infty$, this conserved quantity is simply $\kappa^{2} \gamma^{2} / 4$. Then we have

$$
\rho^{2}+\kappa \gamma \rho=\left|q_{y}\right|^{2},
$$

namely,

$$
1+\kappa \gamma \rho^{-1}=\left|q_{x}\right|^{2} .
$$

Note that Eq. (2.9) can be rewritten into

$$
\rho^{-1} q_{y s}=q+\left(1+\kappa \gamma \rho^{-1}\right) q,
$$

which can be converted into by the conversion relations (2.7).

$$
\partial_{x}\left(\partial_{t}+|q|^{2} \partial_{x}\right) q=q+\left(1+\kappa \gamma \rho^{-1}\right) q .
$$

Finally, we have

$$
\begin{aligned}
q_{x t} & =q-\left(|q|^{2} q_{x}\right)_{x}+\left(1+\kappa \gamma \rho^{-1}\right) q \\
& =q-\left(|q|^{2} q_{x}\right)_{x}+\left|q_{x}\right|^{2} q,
\end{aligned}
$$

which is exactly the defocusing mCSP equation (1.8). 


\subsection{Lax pair}

By scaling transformations

$$
q \rightarrow \frac{1}{2} q, \quad 2 \rho+\kappa \gamma \rightarrow \rho
$$

Eqs. (2.9)-(2.10) become a complex coupled dispersionless equation of defocusing type discussed in [11]. Therefore, we can give the Lax pair for Eqs. (2.9)-(2.10) as follows

$$
\Psi_{y}=U \Psi, \quad \Psi_{s}=V \Psi,
$$

with

$$
\begin{gathered}
U=\mathrm{i} \lambda\left(\begin{array}{cc}
2 \rho+\kappa \gamma & 2 q_{y} \\
-2 q_{y}^{*} & -2 \rho-\kappa \gamma
\end{array}\right), \\
V=\left(\begin{array}{cc}
\frac{\mathrm{i}}{4 \lambda} & -q \\
-q^{*} & -\frac{\mathrm{i}}{4 \lambda}
\end{array}\right) .
\end{gathered}
$$

Based on the hodograph transformation, the Lax pair for the defocusing mCSP equation (1.8) is deduced as

$$
\begin{gathered}
\Psi_{x}=P \Psi, \quad \Psi_{t}=Q \Psi \\
P=\rho^{-1} U=\mathrm{i} \lambda\left(\begin{array}{cc}
1+\left|q_{x}\right|^{2} & 2 q_{x} \\
-2 q_{x}^{*} & -1-\left|q_{x}\right|^{2}
\end{array}\right), \\
Q=-|q|^{2} P+V=\left(\begin{array}{rr}
\frac{\mathrm{i}}{4 \lambda}-\mathrm{i} \lambda|q|^{2}\left(1+\left|q_{x}\right|^{2}\right) & -2 \mathrm{i} \lambda|q|^{2} q_{x}-q \\
2 \mathrm{i} \lambda|q|^{2} q_{x}^{*}-q^{*} & -\frac{\mathrm{i}}{4 \lambda}+\mathrm{i} \lambda|q|^{2}\left(1+\left|q_{x}\right|^{2}\right)
\end{array}\right),
\end{gathered}
$$

whose compatibility condition gives Eq. (1.8).

\subsection{One- and two-dark soliton solutions by Hirota's bilinear method}

Let us find the one-dark soliton solution satisfying the bilinear equations (2.1)-(2.2). To this end, we assume

$$
f=1+e^{2 \eta}, g=1+\alpha e^{2 \eta}, g^{*}=1+\alpha^{*} e^{2 \eta}
$$

where $2 \eta=\beta y+\omega s,|\alpha|=1$. A direct substitution of Eq. (2.23) into the bilinear equation (2.2) yields

$$
2 \omega^{2}=\frac{1}{2}\left(2-\alpha-\alpha^{*}\right)
$$

Letting $\alpha=e^{-2 \mathrm{i} \phi}$, then we may choose $\omega=-\sin \varphi$. 
Substituting Eq. (2.23) into the bilinear equation (2.2), one obtains

$$
\omega \beta(\alpha+1)+\mathrm{i} \omega \kappa(\alpha-1)+\mathrm{i} \gamma \beta(\alpha-1)=0,
$$

thus

$$
\beta=\frac{-\mathrm{i} \omega \kappa(\alpha-1)}{\omega(\alpha+1)+\mathrm{i} \gamma(\alpha-1)}=\frac{-\kappa \sin \varphi}{\cos \varphi-\gamma}
$$

In summary, we have

$$
f=1+e^{2 \eta}, \quad g=1+e^{2(\eta-\mathrm{i} \varphi)},
$$

where

$$
2 \eta=-\sin \varphi\left(s+\frac{\kappa y}{\cos \varphi-\gamma}\right) .
$$

Thus, the single dark soliton can be expressed by

$$
\begin{gathered}
q=\frac{1}{4}\left(\left(1+\mathrm{e}^{-2 \mathrm{i} \varphi}\right)+\left(\mathrm{e}^{-2 \mathrm{i} \varphi}-1\right) \tanh \eta\right) \mathrm{e}^{\mathrm{i}(\kappa y+\gamma s)}, \\
x=-\kappa \gamma y+\frac{1}{4} s+\frac{\sin \varphi \mathrm{e}^{2 \eta}}{1+e^{2 \eta}}, \quad t=s .
\end{gathered}
$$

The non-singularity condition for the single dark soliton is $\rho \neq 0$ for all $(x, t) \in \mathbb{R}^{2}$. To analyze the property of the one-soliton solution, we calculate out

$$
\rho=\frac{\partial x}{\partial y}=-\kappa \frac{\gamma e^{4 \eta}+\left(2 \gamma+\frac{\sin ^{2} \varphi}{\cos \varphi-\gamma}\right) e^{2 \eta}+\gamma}{\left(1+e^{2 \eta}\right)^{2}},
$$

the non-singularity condition is determined by

$$
\left(2 \gamma+\frac{\sin ^{2} \varphi}{\cos \varphi-\gamma}\right)^{2}-4 \gamma^{2}=\frac{\sin ^{2} \varphi\left(\sin ^{2} \varphi+4 \gamma(\cos \varphi-\gamma)\right)}{(\cos \varphi-\gamma)^{2}} .
$$

Therefore, we can define $\Delta=\sin ^{2} \varphi+4 \gamma(\cos \varphi-\gamma)$ and classify this one-dark soliton solution as follows:

- smooth soliton: when $\Delta<0$, the single dark soliton solution is smooth.

- cuspon soliton: when $\Delta=0$, then $\rho$ attains zero at only one point, which leads to a cusponed dark soliton.

- loop soliton: when $\Delta>0$, then $\rho$ attains two zeros, which leads to a looped dark soliton.

The velocity can be found with the following relation and the hodograph transformation

$$
2 \eta=\frac{\sin \varphi}{\gamma(\cos \varphi-\gamma)}\left(x-\gamma(\cos \varphi-\gamma) t-\frac{1}{4} t\right)+c_{1} .
$$

So the velocity of the dark soliton is

$$
v_{s p, 1}=\gamma(\cos \varphi-\gamma)+\frac{1}{4},
$$

and the depth of the trough is $\left.\frac{1}{2}(1-\mid \cos \varphi) \mid\right)$. It should be emphasised here that the one-dark soliton can be a right-moving, left-moving or a stationary one. 


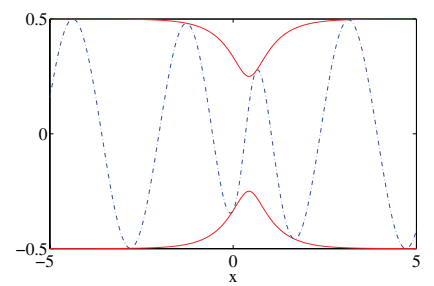

Fig. 1. A smooth dark soliton with parameters $\kappa=1.0, \gamma=0.5, \varphi=2 \pi / 3$ : solid line: $\pm|q|$; dashed line: $\operatorname{Re}(q)$.

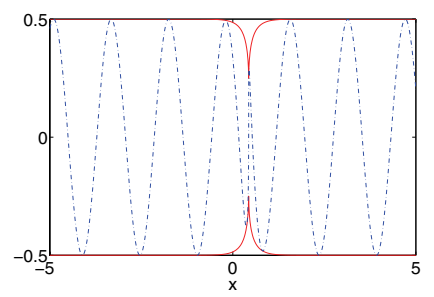

Fig. 2. A cusponed dark soliton with parameters $\kappa=1.0, \gamma=0.25, \varphi=2 \pi / 3$ : solid line: $\pm|q|$; dashed line: $\operatorname{Re}(q)$.

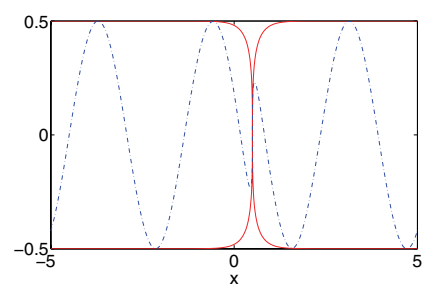

Fig. 3. A cusponed dark soliton with parameters $\kappa=1.0, \gamma=0.5, \varphi=\pi / 2$ : solid line: $\pm|q|$; dashed line: $\operatorname{Re}(q)$.

It is worthy to give further analysis of one-dark soliton solution. Let us first consider how the spatial wave number of background plane waves influences the dark soliton. For the sake of convenience, let us fix the values of $\kappa=1.0, \varphi=2 \pi / 3$, then it can be easily verified that the one-dark soliton solution is a smoothed one for $\gamma>0.25$, a cusponed one for $\gamma=0.25$ and a looped one as $0<\gamma<0.25$. In other words, the localized dark solution has more tendency to become singular (cusponed or looped one) as the spatial wave number of background plane wave increases.

Next, let us look at the influence for the depth of the dark soliton, which is determined by $\frac{1}{2}(1-|\cos \varphi|)$. To this end, we fix the value of $\kappa=1.0, \gamma=0.25$. When $\varphi=2 \pi / 3$, or the depth of 0.25 , the one-dark soliton is a smoothed one. However, when $\varphi=\pi / 2$, or the depth into the maximum value of 0.5 , the dark soliton becomes a cusponed one. So we may conclude that the larger for the depth of the dark soliton, the easier for the formation of the singularity. To illustrate all these fact, we display the profiles of single dark soliton for three set of parameters as in Figs. $1-3$, respectively.

To find two-dark soliton solution, we assume

$$
\begin{aligned}
& f=1+e^{2 \eta_{1}}+e^{2 \eta_{2}}+a_{12} e^{\left(2 \eta_{1}+2 \eta_{2}\right)} \\
& g=1+e^{2\left(\eta_{1}-\mathrm{i} \varphi_{1}\right)}+e^{2\left(\eta_{2}-\mathrm{i} \varphi\right)}+a_{12} e^{2\left(\eta_{1}+\eta_{2}-\mathrm{i} \varphi_{1}-\mathrm{i} \varphi_{2}\right)},
\end{aligned}
$$




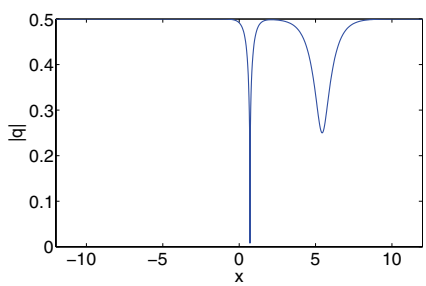

Fig. 4. Smooth-cuspon dark soliton interaction at $t=-20$.

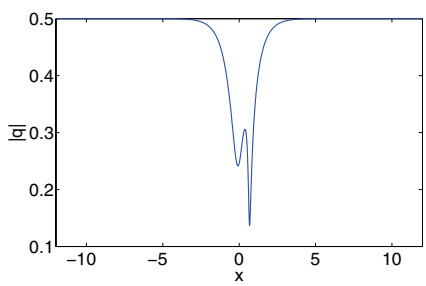

Fig. 5. Smooth-cuspon dark soliton interaction at $t=0$.

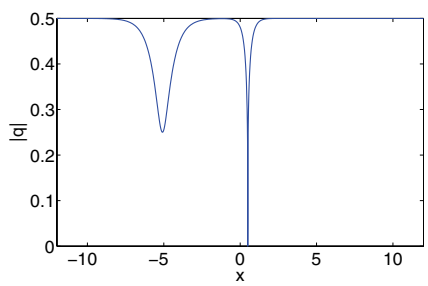

Fig. 6. Smooth-cuspon dark soliton interaction at $t=20$.

where

$$
2 \eta_{i}=-\sin \varphi_{i}\left(s+\frac{\kappa y}{\cos \varphi_{i}-\gamma}\right)
$$

By substituting into the bilinear equation (2.2) and collecting the terms of $e^{2\left(\eta_{1}+\eta_{2}\right)}$, we obtain

$$
a_{12}=\frac{\sin ^{2}\left(\frac{\varphi_{2}-\varphi_{1}}{2}\right)}{\sin ^{2}\left(\frac{\varphi_{2}+\varphi_{1}}{2}\right)} .
$$

The collision processes between smooth-cuspon dark solitons are illustrated in Figs. $4-6$ at $t=$ $-20,0,20$, respectively. The parameters are taken as $\kappa=1.0, \gamma=0.5, \varphi_{1}=\pi / 2, \varphi_{2}=2 \pi / 3$, thus, one is a stationary cusponed soliton, the other is a smoothed soliton with velocity -0.25 . It is seen that the interactions between dark solitons are elastic, which is the same as the collision between dark solitons in defocusing complex short pulse equation and the nonlinear Schrödinger (NLS) equation. Moreover, it seems that if the parameters guarantees the smooth property of each dark soliton, then two dark soliton remains smooth all the time. 


\section{Reductions from the extended KP hierarchy}

The KP-hierarchy reduction method was firstly developed by the Kyoto school [15, 29], and later was used to obtain soliton solutions in the NLS equation, the modified KdV equation, the DaveyStewartson equation and the coupled higher order NLS equations [13,23]. Recently, this method has been applied to derive dark-dark soliton solution in two-coupled NLS equation of the mixed type [24]. In compared to the Hirota's bilinear method [14], the KP-hierarchy reduction method starts with the general KP hierarchy including the two-dimensional Toda-hierarchy [22] and derives the general soliton solution in either determinant or pfaffian form reduced directly from the tau functions of the KP hierarchy. By applying this method, general dark-dark soliton solution was derived in a two-component NLS equation with the focusing-defocusing coupling [24]. Recently, one of the authors [9] has constructed general bright-dark $N$-soliton solution to the vector NLS equation of all possible combinations of nonlinearities including all focusing, all-defocusing and mixed types.

\subsection{Bilinear equations for the extended KP hierarchy}

Let us start with a concrete form of the Gram determinant expression of the tau functions for extended KP hierarchy with negative flows

$$
\tau_{n k l}=\left|m_{i j}^{n k l}\right|_{1 \leq i, j \leq N},
$$

where

$$
\begin{gathered}
m_{i j}^{n k l}=\delta_{i j}+\frac{1}{p_{i}+\bar{p}_{j}} \varphi_{i}^{n k l} \psi_{j}^{n k l} \\
\varphi_{i}^{n k l}=p_{i}^{n}\left(p_{i}-a\right)^{k}\left(p_{i}-b\right)^{l} e^{\xi_{i}}, \quad \psi_{j}^{n k l}=\left(-\frac{1}{\bar{p}_{j}}\right)^{n}\left(-\frac{1}{\bar{p}_{j}+a}\right)^{k}\left(-\frac{1}{\bar{p}_{j}+b}\right)^{l} e^{\bar{\xi}_{j}}
\end{gathered}
$$

with

$$
\begin{aligned}
& \xi_{i}=\frac{1}{p_{i}} x_{-1}+p_{i} x_{1}+\frac{1}{p_{i}-a} t_{a}+\frac{1}{p_{i}-b} t_{b}+\xi_{i 0}, \\
& \bar{\xi}_{j}=\frac{1}{\bar{p}_{j}} x_{-1}+\bar{p}_{j} x_{1}+\frac{1}{\bar{p}_{j}+a} t_{a}+\frac{1}{\bar{p}_{j}+b} t_{b}+\bar{\xi}_{j 0} .
\end{aligned}
$$

Here $p_{i}, \bar{p}_{j}, \xi_{i 0}, \bar{\xi}_{j 0}, a, b$ are constants. Based on the Sato theory for the KP hierarchy [15,29], the above tau functions satisfy a set of bilinear equations

$$
\begin{gathered}
\left(\frac{1}{2} D_{x_{1}} D_{x_{-1}}-1\right) \tau_{n k l} \cdot \tau_{n k l}=-\tau_{n+1, k l} \tau_{n-1, k l}, \\
\left(a D_{t_{a}}-1\right) \tau_{n+1, k l} \cdot \tau_{n k l}=-\tau_{n+1, k-1, l} \tau_{n, k+1, l}, \\
\left(D_{x_{1}}\left(a D_{t_{a}}-1\right)-2 a\right) \tau_{n+1, k l} \cdot \tau_{n k l}=\left(D_{x_{1}}-2 a\right) \tau_{n+1, k-1, l} \cdot \tau_{n, k+1, l}, \\
\left(D_{x_{1}}\left(b D_{t_{b}}-1\right)-2 b\right) \tau_{n+1, k l} \cdot \tau_{n k l}=\left(D_{x_{1}}-2 b\right) \tau_{n+1, k, l-1} \cdot \tau_{n k, l+1} . \\
\text { Co-published by Atlantis Press and Taylor \& Francis } \\
\text { Copyright: the authors } \\
203
\end{gathered}
$$


The proof is given below by referring to the Grammian technique [14,22]. It is easily shown that $m_{i j}^{n k l}, \varphi_{i}^{n k l}, \psi_{j}^{n k l}$ satisfy

$$
\begin{gathered}
\partial_{x_{1}} m_{i j}^{n k l}=\varphi_{i}^{n k l} \psi_{j}^{n k l}, \quad \partial_{x_{-1}} m_{i j}^{n k l}=-\varphi_{i}^{n-1, k l} \psi_{j}^{n+1, k l}, \\
\partial_{t_{a}} m_{i j}^{n k l}=-\varphi_{i}^{n, k-1, l} \psi_{j}^{n, k+1, l}, \\
m_{i j}^{n+1, k l}=m_{i j}^{n k l}+\varphi_{i}^{n k l} \psi_{j}^{n+1, k l}, \quad m_{i j}^{n, k+1, l}=m_{i j}^{n k l}+\varphi_{i}^{n k l} \psi_{j}^{n, k+1, l} .
\end{gathered}
$$

Therefore the following differential and difference formulae hold for $\tau_{n k l}$,

$$
\begin{aligned}
& \partial_{x_{1}} \tau_{n k l}=\left|\begin{array}{cc}
m_{i j}^{n k l} & \varphi_{i}^{n k l} \\
-\psi_{j}^{n k l} & 0
\end{array}\right|, \quad \partial_{x_{-1}} \tau_{n k l}=\left|\begin{array}{cc}
m_{i j}^{n k l} & \varphi_{i}^{n-1, k l} \\
\psi_{j}^{n+1, k l} & 0
\end{array}\right|, \\
& a \partial_{t_{a}} \tau_{n k l}=\left|\begin{array}{cc}
m_{i j}^{n k l} & a \varphi_{i}^{n, k-1, l} \\
\psi_{j}^{n, k+1, l} & 0
\end{array}\right|, \quad \tau_{n+1, k l}=\left|\begin{array}{cc}
m_{i j}^{n k l} & \varphi_{i}^{n k l} \\
-\psi_{j}^{n+1, k l} & 1
\end{array}\right|, \\
& \tau_{n-1, k l}=\left|\begin{array}{ll}
m_{i j}^{n k l} & \varphi_{i}^{n-1, k l} \\
\psi_{j}^{n k l} & 1
\end{array}\right|, \quad \tau_{n, k+1, l}=\left|\begin{array}{cc}
m_{i j}^{n k l} & \varphi_{i}^{n k l} \\
-\psi_{j}^{n, k+1, l} & 1
\end{array}\right| \\
& \tau_{n+1, k-1, l}=\left|\begin{array}{cc}
m_{i j}^{n k l} & a \varphi_{i}^{n, k-1, l} \\
\psi_{j}^{n+1, k l} & 1
\end{array}\right|, \quad \partial_{x_{1}} \tau_{n+1, k l}=\left|\begin{array}{cc}
m_{i j}^{n k l} & \varphi_{i}^{n+1, k l} \\
-\psi_{j}^{n+1, k l} & 0
\end{array}\right|, \\
& \left(\partial_{x_{1}}+a\right) \tau_{n, k+1, l}=\left|\begin{array}{cc}
m_{i j}^{n k l} & \varphi_{i}^{n+1, k l} \\
-\psi_{j}^{n, k+1, l} & a
\end{array}\right|, \\
& \left(\partial_{x_{1}} \partial_{x_{-1}}-1\right) \tau_{n k l}=\left|\begin{array}{ccc}
m_{i j}^{n k l} & \varphi_{i}^{n-1, k l} & \varphi_{i}^{n k l} \\
\psi_{j}^{n+1, k l} & 0 & -1 \\
-\psi_{j}^{n k l} & -1 & 0
\end{array}\right| \\
& \left(a \partial_{t_{a}}-1\right) \tau_{n+1, k l}=\left|\begin{array}{ccc}
m_{i j}^{n k l} & \varphi_{i}^{n k l} & a \varphi_{i}^{n, k-1, l} \\
-\psi_{j}^{n+1, k l} & 1 & -1 \\
\psi_{j}^{n, k+1, l} & -1 & 0
\end{array}\right| \\
& \left(\partial_{x_{1}}\left(a \partial_{t_{a}}-1\right)-a\right) \tau_{n+1, k l}=\left|\begin{array}{ccc}
m_{i j}^{n k l} & \varphi_{i}^{n+1, k l} & a \varphi_{i}^{n, k-1, l} \\
-\psi_{j}^{n+1, k l} & 0 & -1 \\
\psi_{j}^{n, k+1, l} & -a & 0
\end{array}\right| .
\end{aligned}
$$

Applying the Jacobi identity of determinants to the bordered determinants (3.11)-(3.13), the three bilinear equations (3.2)-(3.4) are satisfied. The bilinear equation (3.5) is proved in exactly the same way. 


\subsection{Reductions to the defocusing mCSP equation}

In what follows, we briefly show the reduction processes of reducing bilinear equations of extended KP hierarchy (3.2)-(3.5) to the bilinear equation (2.1)-(2.2). Firstly, we start with dimension reduction by noting that the determinant expression of $\tau_{n k l}$,

$$
\tau_{n k l}=\left|\delta_{i j}+\frac{1}{p_{i}+\bar{p}_{j}} \varphi_{i}^{n k l} \psi_{j}^{n k l}\right|_{1 \leq i, j \leq N},
$$

can be alternatively expressed by

$$
\tau_{n k l}=\left|\delta_{i j}+\frac{1}{p_{i}+\bar{p}_{j}} \varphi_{i}^{n k l} \psi_{i}^{n k l}\right|_{1 \leq i, j \leq N},
$$

by dividing $j$-th column by $\psi_{j}^{n k l}$ and multiplying $i$-th row by $\psi_{i}^{n k l}$ for $1 \leq i, j \leq N$. By taking

$$
\bar{p}_{j}=\frac{1}{p_{j}}, \quad b=-\frac{1}{a},
$$

we can easily check that for $1 \leq i \leq N$

$$
\begin{gathered}
p_{i}+\bar{p}_{i}=\frac{1}{p_{i}}+\frac{1}{\bar{p}_{i}} \\
-a^{2}\left(\frac{1}{p_{i}-a}+\frac{1}{\bar{p}_{i}+a}\right)=\left(\frac{1}{p_{i}-b}+\frac{1}{\bar{p}_{i}+b}\right),
\end{gathered}
$$

and

$$
-\frac{\bar{p}_{i}}{p_{i}}\left(-\frac{p_{i}-a}{\bar{p}_{i}+a}\right)\left(-\frac{p_{i}-b}{\bar{p}_{i}+b}\right)=1,
$$

which implies that $\tau_{n k l}$ satisfies the reduction conditions

$$
\begin{gathered}
\partial_{x_{1}} \tau_{n k l}=\partial_{x_{-1}} \tau_{n k l}, \\
-a^{2} \partial_{t_{a}} \tau_{n k l}=\partial_{t_{b}} \tau_{n k l}, \\
\tau_{n-1, k+1, l+1}=\tau_{n k l} .
\end{gathered}
$$

Therefore the bilinear equation (3.2) is reduced to

$$
\left(\frac{1}{2} D_{x_{1}}^{2}-1\right) \tau_{n k l} \cdot \tau_{n k l}=-\tau_{n+1, k l} \tau_{n-1, k l}
$$

Moreover, by referring to the bilinear equation (3.5) and the reduction conditions (3.16)-(3.17), we have

$$
\left(D_{x_{1}}\left(a D_{t_{a}}-1\right)+2 \frac{1}{a}\right) \tau_{n+1, k l} \cdot \tau_{n k l}=\left(D_{x_{1}}+2 \frac{1}{a}\right) \tau_{n, k+1, l} \cdot \tau_{n+1, k-1, l}
$$


Adding to (3.4), we have

$$
\left(D_{x_{1}}\left(a D_{t_{a}}-1\right)-a+\frac{1}{a}\right) \tau_{n+1, k l} \cdot \tau_{n k l}=\left(-a+\frac{1}{a}\right) \tau_{n+1, k-1, l} \tau_{n, k+1, l}
$$

By using (3.3), one obtains

$$
\left(D_{x_{1}}\left(a D_{t_{a}}-1\right)-a+\frac{1}{a}\right) \tau_{n+1, k l} \cdot \tau_{n k l}=\left(a-\frac{1}{a}\right)\left(a D_{t_{a}}-1\right) \tau_{n+1, k l} \cdot \tau_{n k l},
$$

or

$$
\left(D_{x_{1}}\left(a D_{t_{a}}-1\right)-\left(a^{2}-1\right) D_{t_{a}}\right) \tau_{n+1, k l} \cdot \tau_{n k l}=0 .
$$

Next, we proceed to the reduction of complex conjugate, which turns out to be very simple. Specifically, by taking $a$ pure imaginary, $\left|p_{i}\right|=1$ and $\bar{\xi}_{j 0}=\xi_{j 0}^{*}$, where * means complex conjugate, we have $\bar{p}_{i}=p_{i}^{*}$ and

$$
\tau_{n 00}^{*}=\tau_{-n, 00}
$$

Due to the relation (3.15) and (3.16), $x_{-1}=0$ and $t_{b}=0$ become dummy variables. Therefore, we have

$$
\tau_{n 00}=\left|\delta_{i j}+\frac{1}{p_{i}+p_{j}^{*}}\left(-\frac{p_{i}}{p_{j}^{*}}\right)^{n} e^{\xi_{i}+\xi_{j}^{*}}\right|_{1 \leq i, j \leq N},
$$

with

$$
\xi_{i}=p_{i} x_{1}+\frac{1}{p_{i}-a} t_{a}+\xi_{i 0}
$$

In summary, by defining

$$
f=\tau_{000}, \quad g=\tau_{100}
$$

we arrive at

$$
\begin{gathered}
\left(D_{x_{1}} D_{t_{a}}-\frac{1}{a} D_{x_{1}}-\left(a-\frac{1}{a}\right) D_{t_{a}}\right) g \cdot f=0, \\
\left(\frac{1}{2} D_{x_{1}}^{2}-1\right) f \cdot f=-g g^{*} .
\end{gathered}
$$

Finally, by setting $a=\mathrm{i} c, t_{a}=\kappa c y, x_{1}=s / 2$ and $\left(c^{2}+1\right)=-2 \gamma c$, the above bilinear equations coincide with the bilinear equations (2.1)-(2.2). Therefore, the reduction process is complete. In summary, we provide the determinant solution to the defocusing mCSP equation (1.8) by the following theorem. 
Theorem 3.1. The defocusing $m$ CSP equation (1.8) admits the following determinant solution

$$
q=\frac{1}{2} \frac{g}{f} e^{\mathrm{i}(\kappa y+\gamma s)}, \quad x=-\gamma y+\frac{1}{4} s-(\log f)_{s} \quad t=s,
$$

where

$$
\begin{aligned}
& f=\left|\delta_{i j}+\frac{1}{p_{i}+p_{j}^{*}} e^{\xi_{i}+\xi_{j}^{*}}\right|_{1 \leq i, j \leq N}, \\
& g=\left|\delta_{i j}+\left(-\frac{p_{i}}{p_{j}^{*}}\right) \frac{1}{p_{i}+p_{j}^{*}} e^{\xi_{i}+\xi_{j}^{*}}\right|_{1 \leq i, j \leq N},
\end{aligned}
$$

with

$$
\left|p_{i}\right|=1, \quad \xi_{i}=\frac{\kappa c}{p_{i}-\mathrm{i} c} y+\frac{1}{2} p_{i} s+\xi_{i 0}
$$

under a constraint

$$
\gamma=-\frac{c^{2}+1}{2 c}
$$

By taking $p_{i}=-\mathrm{i} e^{-\mathrm{i} \varphi_{i}}$, one can easily obtain the one- and two-dark soliton solutions to the defocusing mCSP equation (1.8), which coincide the soliton solutions obtained in previous section by Hirota's perturbation method.

\section{Comments and concluding remarks}

In [11], a defocusing CSP equation was derived from physical context in nonlinear optics as an analogue of the NLS equation in ultra-short pulse regime. Its geometric property and multi-dark soliton solution via Hirota's bilinear method was further studied in [12]. In the present paper, we study a modified CSP equation of defocusing type via Hirota's bilinear method. Firstly, we give its bilinear form and found the one- and two-dark soliton solutions, whose properties are investigated in details. Secondly, starting from a set of bilinear equations, along with their tau functions, of a single-component extended KP hierarchy, we derive the defocusing mCSP equation based on the KP-hierarchy reduction method. Meanwhile, its multi-soliton solutions is provided in determinant form. We should comment here that, even if the dark soliton solutions look similar for the CSP and the modified CSP equations, there are actually some fundamental differences between them. For example, keeping all other parameters the same, a cusponed soliton (singularity) is developed for the CSP equation with a background plane wave of amplitude 1.22, whereas, a background plane wave of amplitude 0.5 leads to a cusponed solution. In other words, a singularity is easier to be developed for the modified CSP equation than the CSP equation.

Even though various solutions to the focusing and defocusing mCSP equation have been constructed including the dark soliton solutions in the present paper [21], we should consider a coupled 
modified complex short pulse equation of mixed focusing and defocusing nonlinearity

$$
\begin{aligned}
& q_{1, x t}=q_{1}+q_{1}^{*}\left(\sigma_{1} q_{1} q_{1, x}+\sigma_{2} q_{2} q_{2, x}\right)_{x}, \\
& q_{2, x t}=q_{2}+q_{2}^{*}\left(\sigma_{1} q_{1} q_{1, x}+\sigma_{2} q_{2} q_{2, x}\right)_{x},
\end{aligned}
$$

where $\sigma_{i}= \pm 1$ with 1 represents focusing case and -1 stands for defocusing case. To find all kinds of solutions including the multi-bright, multi-dark soliton and multi-rogue wave solutions and to compare with the results with the ones to the coupled complex short pulse equation will be interesting.

\section{Acknowledgments}

This work is partially supported by the national natural science foundations of China (Grant Nos.11428102, 11371323). The work of YO is partially supported by JSPS Grant-in-Aid for Scientific Research (B-24340029, C-15K04909) and for Challenging Exploratory Research (26610029).

\section{References}

[1] R. Beals, M. Rabelo and K. Tenenblat, Bäcklund transformation and inverse scattering solutions for some pseudospherical surface equations, Stud. Appl. Math.81 (1989) 125-151.

[2] J. C. Brunelli, The short pulse hierarchy, J. Math. Phys. 46 (2005) 123507.

[3] J. C. Brunelli, The bi-Hamiltonian structure of the short pulse equation, Phys. Lett. A 353 (2006) 475478.

[4] Y. Chung, C. K. R. T. Jones, T. Schäfer and C. E. Wayne, Ultra-short pulses in linear and nonlinear media, Nonlinearity 18 (2005) 1351-1374.

[5] A. Dimakis, F. Müller-Hoissen, Bidifferential calculus approach to AKNS hierarchies and their solutions, SIGMA 6 (2010) 055.

[6] B.-F. Feng, K. Maruno, Y. Ohta, Integrable discretization of the short pulse equation,J. Phys. A: Math. Theor. 44 (2010) 085203.

[7] B.-F. Feng, J. Inoguchi, K. Kajiwara, K. Maruno and Y. Ohta, Discrete integrable systems and hodograph transformations arising from motions of discrete plane curves, J. Phys. A: Math. Theor. 44 (2011) 395201.

[8] B.-F. Feng, An integrable coupled short pulse equation, J. Phys. A: Math. Theor. 45 (2012) 085202.

[9] B.-F. Feng, General $N$-soliton solution to a vector nonlinear schrödinger equation. J. Phys. A: Math. Theor. 47 (2014) 355203.

[10] B.-F. Feng, Complex short pulse and coupled complex short pulse equations, Physica D 297 (2015) 085202 .

[11] B.-F. Feng, L. Ling and Z. Zhu, A defocusing complex short pulse equation and its multi-dark soliton solution by Darboux transformation, Phys. Rev. E 93 (2016) 052227.

[12] B.-F. Feng, K. Maruno, Y. Ohta, Geometric formulation and multi-dark solition solution to the defocusing complex short pulse equation, accepted by Stud. Appl. Math..

[13] C. Gilson, J. Hietarinta, J. Nimmo and Y. Ohta, Sasa-Satsuma higher-order nonlinear Schrödinger equation and its bilinearization and multisoliton solutions, Phys. Rev. E 68 (2003) 016614.

[14] R. Hirota, The direct method in soliton theory, (Cambridge University Press, Cambridge, 2004).

[15] M. Jimbo and T. Miwa, Solitons and infinite-dimensional Lie algebras, Publ. RIMS, Kyoto Univ. 19 (1983) 943-1001.

[16] V. K. Kuetche, S. Youssoufa and T. C. Kofane, Ultrashort optical waveguide excitations in uniaxial silica fibers: Elastic collision scenarios, Phys. Rev. E 90 (2014) 063203.

[17] L. Ling, B.-F. Feng and Z. Zhu, Multi-soliton, multi-breather and higher order rogue wave solutions to the complex short pulse equation, Physica D 327 (2016) 13-29. 
[18] Y. Matsuno, Bilinear Transformation Method (Academic Press, New York, 1984).

[19] Y. Matsuno, Multisoliton and multibreather solutions of the short pulse model equation, J. Phys. Soc. Jpn. 76 (2007) 084003.

[20] Y. Matsuno, A novel multi-component generalization of the short pulse equation and its multisoliton solutions, J. Math. Phys. 52 (2011) 123702.

[21] Y. Matsuno, Integrable multi-component generalization of a modified short pulse equation, J. Math. Phys. 57 (2016) 111507.

[22] S. Miyake, Y. Ohta and J. Satsuma, A representation of solutions for the KP hierarchy and its algebraic structure, J. Phys. Soc. Jpn. 59 (1990) 48-55.

[23] Y. Ohta, Wronskian solutions of soliton equations, RIMS Kokyuroku 684 (1989) 1 [In Japanese].

[24] Y. Ohta, D.-S. Wang and J. Yang, General N-dark-dark solitons in the coupled Nonlinear Schrödinger equations, Stud. Appl. Math. 127 (2011) 345-371.

[25] M. L. Robelo, On equations which describe pseudospherical surfaces, Stud. Appl. Math. 81 (1989) 221-248.

[26] A. Sakovich and S. Sakovich, The short pulse equation is integrable, J. Phys. Soc. Jpn. 74 (2005) 239241.

[27] A. Sakovich and S. Sakovich, Solitary wave solutions of the short pulse equation, J. Phys. A: Math. Gen. 39 (2006) L361-L367.

[28] S. Sakovich, Transformation and integrability of a generalized short pulse equation, Commmu. Nonlinear Sci. Numer. Simulat. 39 (2016) 21-28.

[29] M. Sato and Y. Sato, Soliton equations as dymamical systems on infinite dimensional Grassmann manifold, in Nonlinear PDE in Applied Science, U.S.-Japan Seminar, Tokyo, Lecure Notes in Num. Appl. Anal. 5 259-271 (North-Holland, New York, 1983).

[30] T. Schäfer and C. E. Wayne, Propagation of ultra-short optical pulses in cubic nonlinear media, Physica D 196 (2004) 90-105.

[31] S. Shen, B.-F. Feng and Y. Ohta, From the real and complex coupled dispersionless equations to the real and complex short pulse equations, Stud. Appl. Math. 136 (2016) 64-88. 